\title{
Complexity theories: a historic glance
}

\section{Massimiliano Ruzzeddu', Vasja Roblek ${ }^{2}$ \\ mruzzed@hotmail.com}

1) Tenured Researcher in Sociology,

University Niccolò Cusano in Rome.

2) Faculty of Organization Studies in Novo Mesto.

\begin{abstract}
Since their foundation, Complexity theories have been characterized by a wide multiplicity of interpretations, so that it is often hard to find even a commonly shared definition. This work provides a description of the main interpretations of Complexity and gives an account of the differences, in terms of heuristic potentialities, that have not permitted the assessment of a unique definition.

The exploration of these differences will take place within an historical framework. The main idea of this work is that each different version of Complexity Theories is related to the social and cultural structure that characterizes a given epoch.

More specifically, we will try to insert each specific version of CT in a given historical contents and will highlight the socialepistemic needs (which change at each epoch) that that version is supposed to have met.

We hope that this work will bring two categories of benefits: on one side it will highlight the different interpretations and help
\end{abstract}

* Keywords: populism, italian politics, political culture.. 
create a metahistorical notion of Complexity. On the other side, this work might shed light on the cultural differences that have characterized the last decades in scientific communities and in public opinion.

\section{Approaches for systemic science}

In his work "La place du désordre," Boudon states that the notion of complexity is not complex, as the notion or circle is not round. Nevertheless few ideas have shown a higher complexity than "complexity" itself. As a matter of fact,, since Complexity Theories arose in the intellectual scenario, many different points of view have emerged on what Complexity is, how it can affect scientific activity and, more generically, the knowledge process that underpins it.

Thus, although Complexity theories still represent themselves as a "new" discipline, maybe the moment has arrived to insert this discipline in a historical framework, not only for the purpose of intellectual completeness, but especially for shedding light on a scientific matter that has turned to be quite confusing in the last few decades.

Within this framework, we will use Ashby's historical approach (1956: 98), to clarify all the possible nuances of the notion of Complexity instead of a mere descriptive approach. More specifically, we will consider the cultural-social environments of the scientific communities that have created the notion of Complexity (see Lepskyi, 2018; 257). As Kuhn demonstrates, science performances - objects, methodologies, results - largely depend on the representations that, at a given moment, social actors have of science as well as the expectations that science produces. As we are going to see, it is possible to match different representations of Complexity with different models of relationship between theory and reality and different expectations of science.

\section{Complexity theories evolution}

\subsection{The beginning}

Complexity theories are the synthesis of a wide range of studies and experiences that started in the second half of the XX century both in Europe and in the United States. The first step is the foundation of the Systemics Theories during and just after WWII. At the Josiah Macy Jr. Foundation, Frank Fremont-Smith promoted the Macy Conferences for almost two decades from 1941 to 1960 . These conferences were a set of meetings of scholars from various disciplines held in New York, with the explicit aim of promoting meaningful communication across scientific disciplines and restoring unity to science. After the foundations of first order cybernetics were laid, scientists from various fields of science have expanded the scope of cybernetics beyond the control of feedback (Dominici \& Roblek, 2016). In the field of cybernetics there are a large number of other studies such as biology and theory of open systems (von Bertalanffy, 1972), information and communication theory (Weaver \& Shannon, 1964), self - organization and regulation (Ashby, 1956), and management and organizational cybernetics (Beer, 1985).

Ludwig von Bertalanffy (1951) developed his General Systems Theory. This theory emerged to overcome the division of science in which physics, chemistry, biology, economics, psychology, sociology and other disciplines individually examined and deepened their own theories, solutions and models that are only useful in a narrow segment (Bertalanffy, 1968, 30 - 31). General systems theory was meant to act as an integrator of various scientific fields (Hammond, 2019). 
The system is defined as a set of interconnected elements (Bertalanffy, 1968, p. 163). We must treat it as a whole, because its behavior depends not only on the characteristics of the elements it consists of, but also on the nature of the connections between them. One says that "a whole is more than a simple sum of individual parts" and that systems have "emergent" properties that occur only when elements interact in interaction. Changing the state of any element in the system affects the states of all other elements in the system, which also means that each element also affects itself. According to Bertalanffy (1968, 163), this reversal of self-influence can be done according to the principle of "feedback" (as in cybernetic systems) or according to more general and primitive principles of dynamic alignment between all elements (e.g. in neural networks).

Boulding (1956) is also a reference for Systemics. In his article 'General systems theory: The skeleton of science', Boulding proposed a classification of the system in terms of nine hierarchical levels. Levels 1 to 3 contain non-life systems, such as closed physical systems. Biological and open systems are located between 4 and 6 levels. Levels 7 and 8 cover social and behavioral sciences. Here the human being with the social systems is in the foreground. In this case, the systems are open. The last, ninth, level includes religion, theology and philosophy.

Their main feature was that the epistemic and theoretical background of classical science, based upon linear dynamics, had come out to be unfit any longer for a deep and exhausting comprehensions of a larger and larger number of objects, so that they implied defining and testing new notions.

Von Bertalanffy's work is also pivotal for another reason: it contains long discussions on what systems are and what concepts they can include. This is why in the works that are considered the foundations of complexity-related disciplines, very few mention new empirical facts, for those works focus on the definitions of formal concepts. The references to reality are mainly about objects that the readers are likely to have already known.

This is a choice that Von Bertalanffy also clearly assesses: "In the gamut of modern science and life new conceptualizations, new ideas and new categories are required" (Von Bertalanffy, 1968: 10) and "the point to be reiterated is that problems previously not envisaged, not manageable or considered as being beyond science or purely philosophical are progressively explored" (Ibidem: 23). The author only refers to phenomena that the scientific community already knew, such as "Atoms, molecules, crystals, biological structures (...), clocks, conventional machines in general, solar systems (...), symbolism, past and future, self and world, self-awareness etc. (...) populations of organisms (humans included) (Ibidem 28-9). The same trend emerges in other parts of the work: Von Bertalanffy' s goal is to highlight the domains and the principles of systemic, so that he does not report new data or discoveries; on the contrary, the works, which he mentions, are often decades older that the book "General system theory" itself, so that their content is known to most readers or, at least, most readers should know how to deal with that information. In other words, that book shows two different dimensions, crossing each other; on one side, the main objects are the formal systemic notions, such as equifinality or hierarchy etc.; on the other side, Von Bertalanffy shows well-known information in order to insert that into the systemic framework, and have the reader consider those notions from this new scope.

For example, everybody knows and knew, that human bodies swap energy and materials with the external: food, gases etc.; Von Bertalanffy writes, that "The organism is not a closed, but an open system" (Ibidem, 121)

Furthermore, we term a system "closed" if no material enters or leaves it; it is called "open" if there is import and export of material. There is, therefore, a fundamental contrast 
between chemical equilibria and metabolizing organisms. The organism is not a static system closed to the outside and always containing identical components; it is an open system in a (quasi-steady state, maintained constant in its mass relations in a continuous change of component material and energies, in which material continually enters from, and leaves into, the outside environment" (Ibidem: 121).

It is clear that the author has the reader consider all the physiological phenomena that permit the body's survival, and on which any average reader has sufficient knowledge. By showing those notions from the scope of homeostatic equilibrium, this operation will permit the readers to construct a formal and abstract representation of homeostatic equilibrium in their minds.

The same about human psychology: Von Bertalanffy's goal, in this domain, is to rearrange the set of psychological notions, commonly known at his time, within a systemic framework; namely, his idea is to overcome reductionist representations of the human mind, especially the behaviorist, whose authors think that it is possible to explain every human action by the Stimulus-Response equation (Ibidem: 105 and ff.). So that, for describing the systemic approach to psychology, he refers to the main humanistic models, which were popular at that time, and describes the narrowness of their epistemic bases (Ibidem: 188 and ff.). The same approach is evident, when he shows the systemic approach to understand human nature and behavior. "Juvenile delinquents who commit crime for fun, a new psychopathology resulting from too much leisure, the fifty percent metal cases in our hospitals - all this is proof that the scheme of adaptation, adjustment, conformity, psychological and social equilibrium doesn't work. There is a wide range of behavior - and, presumably also of evolution which cannot be reduced to utilitarian principles of adaptation of the individual and survival of the species. Greek sculpture, Renaissance painting, and German music - indeed, any aspect of culture - has nothing to do with utility, or with the better survival of individuals or nations. Mr. Babbit is in every utilitarian respect better off than Beethoven or Michelangelo" (Ibidem191-92). It is the same model for historical theory: in order to match systemics and a nomothetic idea of history he quotes "the Italian philosopher Vico in the early 18th century, and continued in the philosophical systems and the investigations by Hegel, Marx, Spengler, Toynbee, Sorokin, Kroeber and others" (Ibidem: 198). Similarly, in order to demonstrate that the "great civilisations" imply a unified and coherent symbolic system (Ibidem: 201), he relies on the reader's knowledge of the Greek-Roman Asian cultures, as well as the Canadian biculturalism. (Ibidem: 202)

The same trend it is possible to find in another milestone of complex science: "Introduction to cybernetics".

In this work, Ashby provides an example of a different state system by reporting the description of the threespine stickleback coupling "The male's first reaction, the zigzag dance, is dependent on a visual stimulus from the female, in which the sign stimuli "swollen abdomen" and the special movements play a part. The female reacts to the red colour of the male and to his zigzag dance by swimming right towards hi. This movement induces the male to turn round and swim rapidly to the nest. This, in turn, entices the female to follow him, thereby stimulating the male to point its head into the entrance. His behavior now releases the female's next reaction: she enters the nest (...). This again releases the quivering reaction in the male, which induces spawning. The presence of fresh eggs in the nest makes the male fertilize them" (Ashby, 1956: 26; contains quotations from Tinbergern, 1951).

Still, he recalls chemistry related notions to show the notion of "very large systems"; those are systems consisting of a large number of components, that trigger emergent properties by coupling to each other; in order to show that at a microscopic level he fact 
that some coupling does not take place he refers to the creation of $\mathrm{AgCl}$ (silver nitrate in a solution of sodium chloride), that always occurs at a macroscopic level in spite of those local irregularities (Ibidem: 68).

Later in the same book, Ashby provides a vivid example of a cat chasing a mouse; by considering the mouse's conditions (alive, running, harmed, dead etc.) As different states it matches those states with the formal notion of stability (Ibidem 197).

Still in the cybernetic domain, Wiener $(1961,1965)$ shows the same relationship between theory and reality.

Just as a matter of an example, when he needs to show what irreversibility is and how it changes the classical notion of time and order, he refers to the intuitional differences between the order of the planets and the disorder of the clouds.

In the first place, the meteorological system is one involving a vast number of approximately equal particles, some of them very closely coupled to one another, while the astronomical system of the solar universe contains only a relatively small number of particles, greatly diverse in size, and coupled with one another in a sufficiently loose way that the second-order coupling effects do not change the general aspect of the picture we observe, and the very high order coupling effects are completely negligible (...). The position, velocities, and masses of the bodies of the solar system are extremely well known at any time, and the computation of their future and past positions, while not easy in detail, is easy and precise in principle. On the other hand, in meteorology, the number of particles concerned is so enormous that an accurate record of their initial positions and velocities is utterly impossible (Wiener, 1961: 30 -31).

No need to highlight that, in that passage, the reader can find it more difficult to catch the meaning of the notions of second-order or high-order coupling that figure out a meteorological system or the planets around the sun.

It is possible to mention many other examples like this, though, generally speaking, we can assess that the first period of Complexity runs from the mid 1940s through the late 1960s. This was a period when the main notions related to Complexity emerges during long debates among specialists devoted to solve scientific or technical problems.

We have seen that in the first part of their history the classics of complexity principally aimed to formally define the theoretical framework of the domain, as well as its principal notions.

As a consequence, it is possible to assess that the relationship with empirical reality is mainly inductive: the theoretical effort, in this phase is exclusively analytical, for the aim is to provide effective and logical coherent concepts; this means that the references are about parts of reality, that most readers are likely to know or on which they can easily achieve information. The empirical references are principally examples that can show the meaning or the domain of any theoretical discourse.

We can assess, that this phase ends in the late '60, when the main classics, above mentioned, are issued.

\subsection{The $1970 \mathrm{~s}$ and 1980}

The emergence of information technologies in the 1970s led to the introduction of new communication channels between different systems that attempt to control each other. In the period from the early 1970s to the 1980s of the 20th century, second-order cybernetics was developed in response to some epistemic problems in the social sciences, for which firstorder cybernetics has not found answers (Geyer, 1995).

The following phase implies a different relationship between theory and reality; in fact, 
Complexity Theories started being considered a complete set of theoretical statements, and expectations started arising of providing a better knowledge on reality. From this point of view, a distinction is necessary between two research paths related to different domains.

First, Complexity Theories have been the theoretical frameworks for studies on perception; although this field has implied heavy consequences for philosophical and sociological theory, it is an example of the typical inductive-deductive relationship theoryreality in the traditional science; in fact, theoretical debates and studies on perception, which had taken place independently (see Mascolo, 2011, 65 and ff.) were the theoretical base for the gestalt based idea of knowledge; on its turn, this theoretical core orientated studies and empirical researches, which confirmed the hypothesis, that the neuronal system of superior animals does not perceive images from the environment, but only simple inputs; the images that portray the external world, are the outcome of a construction process, which takes place in the nervous system. These empirical findings gave birth to the Second Cybernetics (see Maturana-Varela, 1988) as well as the parts of Piaget $(1967 ; 1970)$ Ceruti (1986) etc; those authors would provide strong references for new visions of the world, based upon the idea of the constructed character of reality; those visions are powerful sense - making instruments, just like classic systemic was; nevertheless, it is interesting to consider that cognitive studies have constitute a classical domain on empirical research, basing upon complex categories.

Second, in the same period other ways of conceiving complexity arose.

Before treating them, it is necessary to assess the social and the cultural condition of the time when those theoretical streams appeared. In facts, we are in the late 70's and, in the Western countries, the era of the new, revolutionary social movements, which had been actual mass phenomena in so many countries, was next to finish.

According to Lyotard's terminology (1979) the political narrations that had orientated the visions-of-the-world and the actions of the baby boomers' generation was losing credibility; fewer and fewer actors believed that a perfect society would arise, in which no injustice, inequality or social constraint would exist any longer; the conviction was mainly fading away, that this perfect society would be the consequence of organized, human (political) actions and, generally, that human reason and would be able to change reality no matter what obstacles would appear.

The late 1970 s are considered the beginning of the era of "disillusion", in which social reality (just like the natural world) appeared to be independent and stronger than human intentions, and market a more effective institution than politics at regulating social relationships. Within this framework, also the social expectations of social and human sciences underwent a strong change: namely, individuals stopped considering social sciences as an instrument to make justice and freedom come true in every society.

Since that moment, social science was supposed to "simply" describe how social reality worked and changed, so that social actors could develop effective strategies of adaptation.

Needless to say, the fact that social structure and changes exist independently from the individual subjects, recalls the structuralist idea of society, that had emerged between late XIX and early XX centuries, in France and USA.

Therefore, in this phase, the epistemic debate among Complexity theorists mainly highlighted the notions that fit to this framework. Typical example is the concept of "emergence": the fact that a set of elementary or simple elements can join together and show collective properties - which none of those units use to have singularly - can perfectly describe a social reality, that seemed so far and independent from human beings; in fact, emerging properties do not fit to traditional logic and science, which base upon linear dynamics and cannot operate prevision on those phenomena, like they did with mechanical properties. 
Even less is the subject able to manipulate or control emerging changes; while in the former decades the human - especially collective - action seemed to be effective at changing the social world, it is now clear that one of the core characters of emerging phenomena, is that they are "social facts": they are independent from human will and actions.

Briefly speaking, the only set of strategies that social actions are able to perform, are the adaptations to this reality. As a consequence, social science is supposed to assess what those strategies are and, if possible, how effective they are.

This is perhaps the reason why Complexity Theories' first wave of popularity took place just in the 1980s: their robust logic coherence, as well as the fact that their rooted in the natural science, made of those theories a valid response to Marxist theories, that still appeared able to put capitalist reality into discussion, although radical movements had become so weak, that they did not represent a threaten any longer. Of course, other notions complexity related notions meet the need to scientifically respond to radical thought: words like "boundary" or "hierarchy", that in the systemic theory have a peculiar meaning, in the sociological and political language might be used as a "naturalistic" response to "scientific" Marxism. Those experiences are related to different theoretical and practical problems (see Heims, 1991) and took place in quite different domains, like physics, engineering, psychology, communication, management etc. This trend is even more evident if we consider the social meaning of the notion "system" at that time: also due to a matter of etymologic closeness, the only kind of system, with which the main authors deal, is the "social system" or, in a broader sense, society itself. In fact, by considering it as the emergence from elementary units, the whole society turns to be the outcome of a process of "self-organisation", i.e. independent from individuals' intentions. Furthermore, this match of structuralism and systemics permitted to overcome the limits of the classical interpretation of structuralism: it is in fact renowned, that the main limits of Durkheim or Parsons' theories was the fact that they had left too little room or the social/historical changes and provided a very static representation of the society. On the contrary, systemics are a theoretical framework that also takes into account the dynamic sides of any society and can include change, evolution and progress (even conflict) and the same time, still portray societies like independent beings. Typical example of this time complexity is Morin's thought.

When he starts reflections about complexity, he does not only highlight the structuralist dimensions of complexity, but also tries to focus on the notions of incertitude. For example, in his work "La Nature de la Nature" (1977) he matches the dimensions in his reflections on the problem of the order: starting from the physical world, he tries to construct an ideal type of the relationship between order and chaos that happen in the reality.

'L'univers ne s'est pas seulement construit malgré le désordre, il s'est aussi construit dans et par le désordre, c'est-à-dire dans et par la catastrophe originaire et les ruptures qui ont suivi, dans et par le déploiement désordonné de chaleur, Le désordre est partout en action. II permet (fluctuations), nourrit (rencontres) la constitution et le développement des phénomènes organisés. II co-organise et désorganise alternativement et en même temps tout le devenir est marqué par le désordre: ruptures, schismes, déviances sont les conditions des créations, naissances, morphogénèses. Rappelons que le soleil, né en catastrophe, mourra en catastrophe. Rappelons que la terre, tout en tournant sagement et régulièrement autour du soleil, a une histoire faite de cataclysmes, effondrements, plissements, éruptions, inondations, dérives, érosions' (Morin, 1977: 75)

Of course, systemics is the theoretical framework for this operation, so that essential notions of appear, such as entropy, emergence etc.; this set of notions also implies reflecting on the limits of the observer and the influences that this observer exercises of the object and the perception process. 
Namely, the doubt is expressed that the lack of order, which the observer perceives, is actual chaos or depends on the observer's incapability to seize a more complex, or subtle order that hides behind the apparent chaos.

In spite of this, in his following words the focus on this problem seems to fade away. He keeps mentioning that issue, as well as the incertitude in the knowledge process, but the sensation is strong that he believes that just mentioning the problem could be enough to overcome it. On the contrary, he strongly insists on the structuralist part of complexity: by matching the notions of system and society, he often claims that a deeper knowledge of social phenomena will be possible (See Morin 1982, 1990).

This perfectly matches with the cultural climate of the 1980s, in which the belief was great to control reality, although a perfect world, in the Marxist way, or according to the Frankfurter idea of Reason: now society is a system and implies boundaries and hierarchies, i.e. inequality, power asymmetries, etc.

The other example of systemics in these years is the Santa Fé Institute, set in the New Mexico's capital.

Like many other experiences in the complexity domain, it bases upon the expertise on systemic studies in the fields of natural sciences.

Though, differently from Morin, the members of Santa Fé Institute never try to ontologically define society and social facts through systemic (See Waldrop, 1994); they just have yielded an original intuition about the relationship with social reality, when they find out that they might use stochastic previsions to foresee the behavior of complex systems like demographic trends and stock markets.

In fact, according to the theoretical grounds of Santa Fé Institute, a system is a set of a large number of units, whose emergence-related features cannot be the object of linear previsions; though it is possible to overcome this incertitude through statistic calculations and simulation processes.

Now, the idea of using systemics to make previsions on the markets, does not only fit to the political and social environments, that were existing in the 80's, when the economic and financial success was the main expectation on social actors; this also implies a peculiar vision of the world: Santa Fé Institute members never talk about global positive changes, i.e. the possibility of making justice and equality make through in a next or remote future; on the other side, the idea of using complex, mathematical categories, at the end of the day to make money, reflects a strong optimism about the possibility of using complexity theories to master these categories of objects like traditional science did with mechanical phenomena.

In the 80 s of the 20th century, cybernetic science also found its way into the theory of management. Thus, Lynch and Kordis (1988) presented complexity theory as a new and important paradigm for corporate development, for which Merry and Kassavin (1995) considered it to be an important organizational tool..

\subsection{The 1990s}

What follows later, is quite complicated to describe; the 1990s are a really complex time, so that finding cultural and intellectual trends, both among scientific communities and the whole societies, is very hard. In fact, the end of the big narrations that had started already in the 1970s, in the 1990s became largely widespread in many layers of Western societies. Nevertheless, this lack of robust references among social actors was quite far from producing anxiety or pessimism; also thanks to good performances of world economy, which was experimenting a boom of e-commerce and web-based economy, social actors were looking at the global changes with a sort of optimism; in fact, the subtle belief was widely spread, the 
collapse of certitudes that was taking place would also imply the triumph over the old cultural traditions and geographical boundaries.

The arising of new gender identities, the mix of different cultures made possible by migrants' integration and the opening of many frontiers and the affordable price of plane tickets. All this gave rise to positive feelings toward the future. We are already very far from the strong and general utopias that prevailed in the 1960s and the 1970s, nevertheless, in large parts of Western societies, the belief was strong that on the individual point of view, this condition of indeterminacy as an offspring of opportunities, at the same time, reduced the need for reliable previsions of the future. This cultural environment does reflect the scientific debate of that time: in fact, on the sociological side, the main authors of those times are Giddens (1990) and Baumann (1993, 2000, 2001), that depicts, with different styles and lexicons, the fast change pace that was occurring and the incertitude that it implied. In this moment, many are the works devoted to complexity and many are the debate trends among scientific communities.

Among the most representatives, there are the works of Prigogine (especially 1996 and 2003), which principally underline the dimension of incertitude that complexity entails.

Generally speaking, we can assess that while the early complexity theorists had based upon the notion of entropy; Prigogine's theory considers the principle of indeterminacy as the main tool for interpretation of reality (Prigogine, 1991: $151 \mathrm{ff})$. Just like entropy, indeterminacy breaks the linear determinism of classical rationality and opens to a world uncertain and changeable.

Of course, this is not only valid in the physical and biological realms, but also in the human and social realities.

What the observers can do is only accepting and managing this incertitude, by basing up statistics or scenarios.

In other words, the debate on complexity in the 1990s was principally focusing on the impossibility of overcoming the incertitude that often characterizes observed phenomena, while the main intellectual effort of the theorists was to yield notions that could at least describe this incertitude, like the of one of bifurcation17 (Ibidem 80) or time arrow (Ibidem, 85 e ff) etc.

Nevertheless, this was far to be a problem from large parts of the public. On the contrary, this general idea of indetermination did fit to the visions of the world of most social actors that claimed for life styles based upon spontaneity and free individual choices; in fact, indetermination is an effective instrument to asses that traditional references, no matter if political, social or economic, are just useless, if not problematic boundaries; traditions, rules, conventions are far from be reliable benchmarks for decision making, because they cannot tackle with the tremendous incertitude, that affects any existence; as a consequence, the only way for every human being to deal with this dimension is to keep free and accept the good and the bad that incertitude implies.

The prefix "Cyber" has become widely used since the early 1990s. The prefix comes from the Wiener's (1948) coinage of "cybernetics" which is derived from the Greek word "kubernetes", which means "the helmsman" in translation. The meaning could also be defined as the art of control. In the primary sense of the word was meant to steer the boat (Bai \& Lindberg, 1999, 793). Despite its interdisciplinary nature, the term cybernetics has been used to describe the technical and engineering approach, which is primarily focused on the investigation of information feedback loops, control systems and the control of intelligent machines (Mesjasz, 2006; Wiener, 1948). With the technological developments from the last decade of 20th century until today, it becomes important for policy - makers, and academic researchers to understand the nature and extent of the "cyber" debate, such as cyberspace, 
cyber security, cybercrime, cyber war, cyber democracy, cyber party, etc. (Shires \& Smeets, 2017).

The 4th industrial revolution has created a cyber-physical environment and the emergence of the smart (intelligent) systems (e.g. smart factory, smart community) that influence organizational changes and adoption. Therefore, it is crucial that the scientists have to explore a living system that has its own "will" regardless of its level of development (technological development, degree of reorganization, leaders and the willingness of management to move from the "old" system to a smart system and cyberspace, self-organized system). Smart living systems have become organic and therefore difficult to handle. Their interactions with their environment are complicated and it is impossible to predict more than one step ahead. It relies on a system of increasing controllability, developed by second-order cybernetics; cybernetics focuses on understanding biological and social complexity and how it is controlled. It is expected that technological development in the coming years will lead to an equal cooperation between technology and people, both in production processes and in social decision making (Roblek, Erenda \& Meško, 2020). This new coexistence between artificial intelligence and humans also opens up a thinking about the cybernetics of the new order, but this thematic goes beyond the purpose of this article.

\section{Conclusion: in the Third Millennium}

Although globalization in the last decade has much strengthened, at least in the Western societies, this has not implied and improvement of life conditions.

International terrorism, financial crisis, unfair income distribution, etc. have produced deep changes in social actors live.

The increased change rate in the material life conditions and the international relationships have turned quite useless the traditional cognitive references, that cultures had provided.

Politics, religion and science have lost credibility among social actors, and even rationality itself is not able any longer to provide reliable representations and previsions of reality.

Though, the real change that has occurred in those last years, is that social actors do not represent any longer this uncertainty as a source of opportunity or as a synonymous of freedom.

Because of the lower life conditions, what social actors seem to need most at the moment is stable and reliable standpoints that can help them face the current turbulences (See, for example, Fröhlich, 2019; Levin 2012: Matthias et al. 2018).

This implies a deep reflection on the role of science and namely, Complexity Theories. For sure, what is to pass by, is that sort of laziness that affected sociology and social science in the 90's. In fact, once that social theory acknowledged that it was impossible to perfectly mirror social phenomena, it principally devoted to describe the reasons and the conditions if this ignorance; although this limitedness also implied that social hetero-direction from above authorities is never completely. 


\section{References}

Ashby, W. R. (1956). An introduction to cybernetics. London, Champman \& Hall.

Bai, G., \& Lindberg, L. A. (1999). A sociocybernetic approach to information systems development. Kybernetes, 28(6/7), 792-809.

Beer, S. (1985). Diagnosing the system for organizations. Chichester, John Wiley \& Sons Inc.

Boulding, K. E. (1956). General systems theory-the skeleton of science. Management science, 2(3), 197-208.

Dominici, G. and Roblek, V. (2016). Complexity theory for a new managerial paradigm: a research framework. In Ragus, Vrdoljak I., Podrug, N. and Jelenc, L. (Ed.), Neostrategic Management: Contributions to Management Science (pp. 223 - 241). Chem, Springer International Publishing AG.

Fröhlich T. (2019). General system theory (GST) and a non reductionist concept of elements: Suggesting a corresponding discussion based on Tramonti. Syst Res Behav Sci.;36

Geyer, F. (1995). The challenge of sociocybernetics. Kybernetes, 24(4), 6-32.

Hammond D. (2019) The legacy of Ludwig von Bertalanffy and its relevance for our time. Syst Res Behav Sci.,36

Heims S. J. (1991) The Cybernetics Group. Cambridge, the MIT Press

Lepskiy V .(2018) Evolution of cybernetics: philosophical and methodological analysis. Kybernetes Vol. 47 No. 2

Levin S. (2012) Social-ecological systems as complex adaptive systems: modeling and policy implications. Environment and Development Economics 18

Lynch, D., \& Kordis, P. (1988). Strategy of the dolphin: Scoring a win in a chaotic world. New York, William Morrow.

Matthias G. W. Et al. (2018) From Nonprofit Diversity to Organizational Multifunctionality: A Systems-Theoretical Proposal. Administration \& Society 2, Vol. 50(7)

Mesjasz, C. (2006). Przegląd teoretycznych koncepcji nadzoru korporacyjnego. Zeszyty Naukowe/Akademia Ekonomiczna w Krakowie, (700), 47-62.

Merry, U., \& Kassavin, N. (1995). Coping with uncertainty: Insights from the new sciences of chaos, selforganization, and complexity. Santa Barbara, CA, Praeger.

Roblek, V., Erenda, I., \& Meško. M. (2020). Fundamental changes in the organisational processes: Industry 4.0 case study. In Machado, C. \& Davim, P.J. (Eds.), Industry 4.0: Challenges, Trends, and Solutions in Management and Engineering, 1st. ed. Boca Raton, CRC Press. In press.

Shires, J., \& Smeets, M. (2017). Contesting 'cyber'. Washington, DC, New America Foundation

Von Bertalanffy, L. (1951). General system theory, a new approach to unity of science. 5. Conclusion. Human biology, 23(4), 337.

Von Bertalanffy, K.L., 1968. General System Theory: Foundations. Development Applications, New York.

Von Bertalanffy, L. (1972). The history and status of general systems theory. Academy of management journal, 15(4), 407-426.

Weaver, W., \& Shannon, C. (1964). The mathematical theory of information. Urbana: Illinois Press.

Wiener, N. (1948). Cybernetics. Paris, Hermann.

Wiener, N. (1965). Cybernetics or control and communication in the animal and the machine. Cambridge, MA, MIT press.

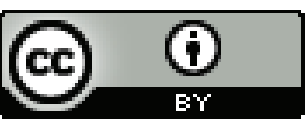

This article is distributed under the terms of the Creative Commons Attribution 4.0 License (https://creativecommons. org/licenses/by/4.0/) which permits any use, reproduction and distribution of the work without further permission provided the original work is attributed as specified on the WCSA Journal by World Complexity Science Academy (https://wcsajournal.com/wcsaj-legal-statement/). 
